\title{
Even resilient doctors report high levels of burnout, finds CMA survey
}

Cite as: CMAJ 2018 October 29;190:E1293. doi: 10.1503/cmaj.109-5674

Posted on cmajnews.com on Oct. 11, 2018.

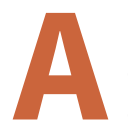
bout a third of Canadian doctors are burned out or depressed, and nearly 1 in 10 have thought about suicide in the past year, but it's not because they lack coping skills, according to a national survey conducted by the Canadian Medical Association (CMA).

Nearly $60 \%$ of doctors said their overall mental health was flourishing, and $82 \%$ reported high levels of resilience. Yet, according to the CMA, "they are increasingly voicing distress and calling for resources and support."

Of the 2547 physicians and 400 medical residents surveyed, $30 \%$ reported high levels of burnout, meaning they experienced symptoms of emotional exhaustion and depersonalization at least weekly. Thirty-four percent met criteria for depression. Nearly one in five reported having thoughts of suicide at some point in their lives; $8 \%$ thought about suicide in the past 12 months.

Medical residents, female physicians, and doctors in their first five years of practice reported the highest rates of burnout, depression and suicidal thoughts. However, these issues cut across the profession, with no significant differences in rates between regions or specialties. This suggests the problem goes beyond any individual's ability to cope.

Most efforts to prevent burnout have focused on improving physicians' personal resiliency rather than their workplaces and training environments. However, according to CMA, "strengthening the health and wellness of the physician workforce is a shared responsibility." While physicians must take steps to maintain their health, "system-level initiatives involving numer-

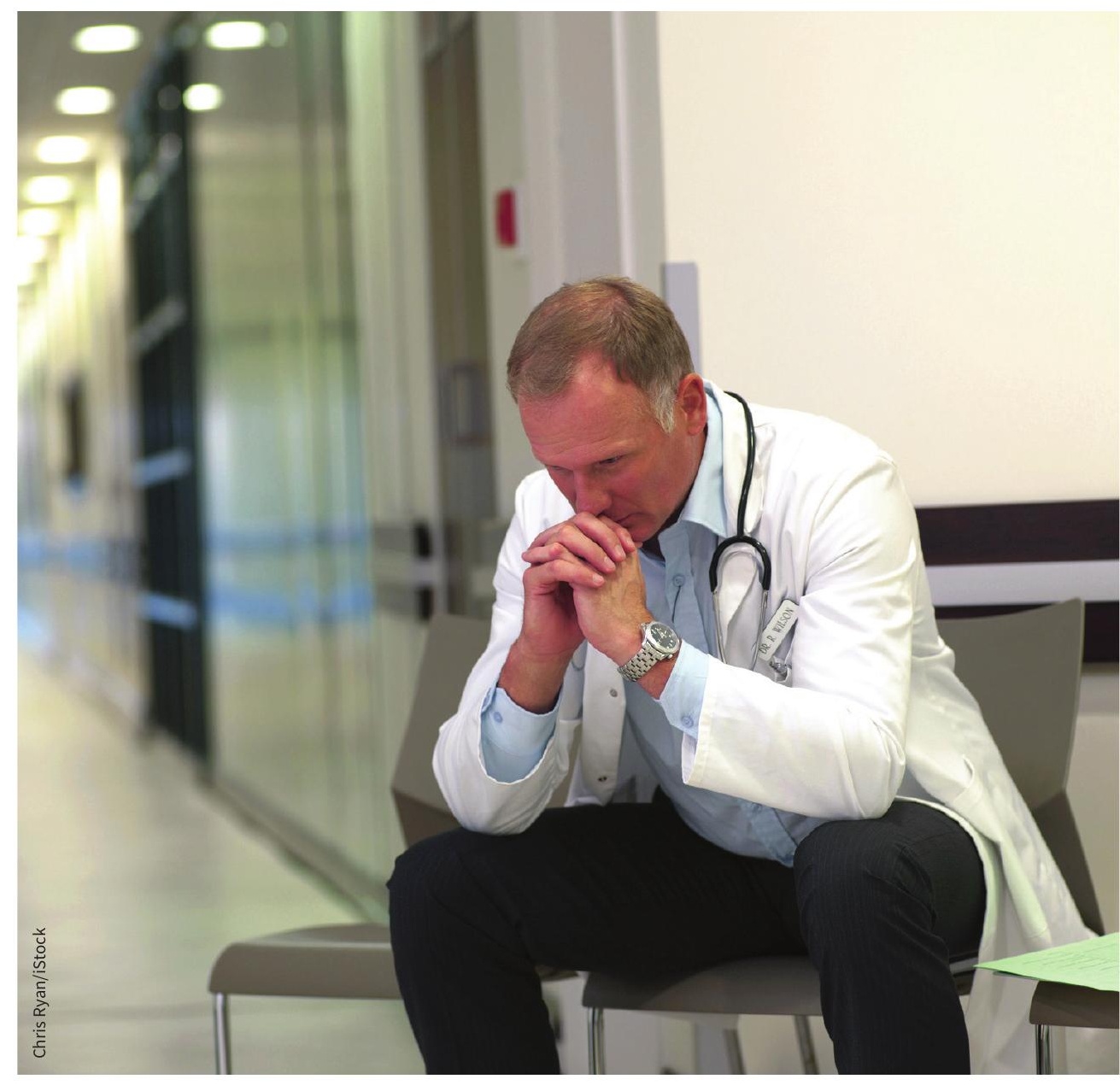

Most doctors are highly resilient but nearly one in three experience symptoms of burnout on a weekly basis.

ous institutions, organizations and communities are also necessary."

The culture of medicine often encourages doctors and trainees to push beyond their limits and discourages seeking help. While $81 \%$ of those surveyed were aware of the physician health services available to them, only $15 \%$ had accessed those supports in the last five years. Top reasons why doctors didn't seek help included shame and believing their situation wasn't severe enough.

Lauren Vogel, CMAJ 